doi: $10.13108 / 2017-9-2-55$

\title{
LOWER BOUNDS FOR THE AREA OF THE IMAGE OF A CIRCLE
}

\author{
B.A. KLISHCHUK, R.R. SALIMOV
}

\begin{abstract}
In the work we consider $Q$-homeomorphisms w.r.t $p$-modulus on the complex plane as $p>2$. We obtain a lower bound for the area of the image of a circle under such mappings. We solve the extremal problem on minimizing the functional of the area of the image of a circle.
\end{abstract}

Keywords: $p$-modulus of a family of curves, $p$-capacity of condenser, quasiconformal mappings, $Q$-homeomorphisms w.r.t. $p$-modulus.

Mathematics Subject Classification: 3065

\section{INTRODUCTION}

The problem on area deformations under quasi-conformal mappings originates from work by B. Bojarskii [1]. A series of results in this direction were obtained in works [2]-4].

First an upper bound for the are of the image of a circle under quasi-conformal mappings was provided in monograph by M.A. Lavrent'ev, see [5]. In [6, Prop. 3.7], the Lavrentiev's inequality was specified in terms of the angular dilatation. Also earlier in works [7]-[8] there were obtained the upper bounds for the area deformation for annular and lower and $Q$-homeomorphisms. In the present work we obtain lower bounds for the area of the image of a circle under $Q$-homeomorphisms w.r.t. $p$-modulus as $p>2$.

To simplify the presentation, we restrict ourselves by the planar case. We recall some definitions. Assume that we are given a family $\Gamma$ of curves $\gamma$ in the complex plane $\mathbb{C}$. A Borel function $\varrho: \mathbb{C} \rightarrow[0, \infty]$ is called admissible for $\Gamma$, which is written as $\varrho \in \operatorname{adm} \Gamma$, if

$$
\int_{\gamma} \varrho(z)|d z| \geqslant 1 \quad \forall \gamma \in \Gamma .
$$

Let $p \in(1, \infty)$. Then a $p$-modulus of the family $\Gamma$ is the quantity

$$
\mathcal{M}_{p}(\Gamma)=\inf _{\varrho \in \operatorname{adm} \Gamma} \int_{\mathbb{C}} \varrho^{p}(z) d m(z) .
$$

Assume that $D$ is a domain in the complex plane $\mathbb{C}$, that is, an open connected subset $\mathbb{C}$ and $Q: D \rightarrow[0, \infty]$ is a measurable function. A homeomorphism $f: D \rightarrow \mathbb{C}$ is called a $Q$-homeomorphism w.r.t. $p$-modulus if

$$
\mathcal{M}_{p}(f \Gamma) \leqslant \int_{D} Q(z) \varrho^{p}(z) d m(z)
$$

for each family $\Gamma$ of curves in $D$ and each admissible function $\varrho$ for $\Gamma$.

B.A. Klishchuk, R.R. Salimov, Lower bounds for the area of the image of a circle.

(c) B.A. Klishchuk, R.R. Salimov. 2017.

Submitted June 16, 2016. 
The study of the inequalities of type (3) as $p=2$ goes back to L. Ahlfors, see, for instance, [9, Ch. I, Sect. D, Thm. 3] as well as to O. Lehto and K. Virtanen [10, Ch. V, Sect. 6.3, Ineq. (6.6)]. In work [11] by C.J. Bishop, V.Ya. Gutlaynskii, O. Martio, M. Vuorinen, a multi-dimensional analogue of inequality (3) was proved for quasi-conformal mappings.

We also note that if the function $Q$ in $(3)$ is bounded almost everywhere by some constant $K \in$ $[1, \infty)$ and $p=2$, then we arrive at classical quasi-conformal mappings introduced originally in works by Grötzsch, Lavrentiev and Morrey.

Let $Q: D \rightarrow[0, \infty]$ be a measurable function. For each number $r>0$ we denote by

$$
q_{z_{0}}(r)=\frac{1}{2 \pi r} \int_{S\left(z_{0}, r\right)} Q(z)|d z|
$$

the integral mean of the function $Q$ over the circle $S\left(z_{0}, r\right)=\left\{z \in \mathbb{C}:\left|z-z_{0}\right|=r\right\}$.

Theorem 1. Let $D$ and $D^{\prime}$ be bounded domains in $\mathbb{C}$ and $f: D \rightarrow D^{\prime}$ be a Q-homeomorphism w.r.t. $p$-modulus, $p>2, Q \in L_{\text {loc }}^{1}\left(D \backslash\left\{z_{0}\right\}\right)$. Then for all $r \in\left(0, d_{0}\right)$, $d_{0}=$ $\operatorname{dist}\left(z_{0}, \partial D\right)$ the esimate

$$
\left|f B\left(z_{0}, r\right)\right| \geqslant \pi\left(\frac{p-2}{p-1}\right)^{\frac{2(p-1)}{p-2}}\left(\int_{0}^{r} \frac{d t}{t^{\frac{1}{p-1}} q_{z_{0}}^{\frac{1}{p-1}}(t)}\right)^{\frac{2(p-1)}{p-2}}
$$

holds true, where $B\left(z_{0}, r\right)=\left\{z \in \mathbb{C}:\left|z-z_{0}\right| \leqslant r\right\}$.

We note that as $p>2$ and $Q(z) \leqslant K$, by Theorem 1 we arrive to the result for a circle in [12, Lm. 7].

\section{ProOF OF MAIN THEOREM}

We provide some auxiliary information about the capacity of a condenser. Following work [13, the pair $\mathcal{E}=(A, C)$, where $A \subset \mathbb{C}$ is an open set and $C$ is a non-empty compact set contained in $A$ is called condenser. A condenser $\mathcal{E}$ is called an annular condenser if $\mathfrak{R}=A \backslash C$ is an annulus, that is, if $\mathfrak{R}$ is a domain whose complement $\overline{\mathbb{C}} \backslash \mathfrak{R}$ consists exactly of two components. A condenser $\mathcal{E}$ is called a bounded condenser if the set $A$ is bounded. We also say that a condenser $\mathcal{E}=(A, C)$ lies in the domain $D$ if $A \subset D$. It is obvious that if $f: D \rightarrow \mathbb{C}$ is a continuous open mapping and $\mathcal{E}=(A, C)$ is a condenser in $D$, then $(f A, f C)$ is also a condenser in $f D$. We also have $f \mathcal{E}=(f A, f C)$.

Let $\mathcal{E}=(A, C)$ be a condenser. By $\mathcal{C}_{0}(A)$ we denote the set of continuous compactly supported functions $u: A \rightarrow \mathbb{R}^{1}$, by $\mathcal{W}_{0}(\mathcal{E})=\mathcal{W}_{0}(A, C)$ we denote the family of non-negative functions $u: A \rightarrow \mathbb{R}^{1}$ such that

1) $u \in \mathcal{C}_{0}(A)$,

2) $u(x) \geqslant 1$ for $x \in C$,

3) $u$ belongs to the class ACL.

As $p \geqslant 1$, the quantity

$$
\operatorname{cap}_{p} \mathcal{E}=\operatorname{cap}_{p}(A, C)=\inf _{u \in \mathcal{W}_{0}(\mathcal{E})} \int_{A}|\nabla u|^{p} d m(z),
$$

where

$$
|\nabla u|=\sqrt{\left(\frac{\partial u}{\partial x}\right)^{2}+\left(\frac{\partial u}{\partial y}\right)^{2}}
$$


is called a $p$-capacity of the condenser $\mathcal{E}$. In what follows we shall make use the identity

$$
\operatorname{cap}_{p} \mathcal{E}=\mathcal{M}_{p}(\Delta(\partial A, \partial C ; A \backslash C))
$$

established in work [14, where for the sets $\mathcal{F}_{1}, \mathcal{F}_{2}$ and $\mathcal{F}$ in $\mathbb{C}$, the symbol $\Delta\left(\mathcal{F}_{1}, \mathcal{F}_{2} ; \mathcal{F}\right)$ stands for the family of all continuous curves connecting $\mathcal{F}_{1}$ and $\mathcal{F}_{2}$ in $\mathcal{F}$.

It is known [15, Prop. 5] that as $p \geqslant 1$,

$$
\operatorname{cap}_{p} \mathcal{E} \geqslant \frac{[\inf l(\sigma)]^{p}}{|A \backslash C|^{p-1}}
$$

Here $l(\sigma)$ is the length of a smooth (infinitely differentiable) curve $\sigma$ being the boundary $\sigma=\partial U$ of a bounded open set $U$ containing $C$ and contained together with its closure $\bar{U}$ in $A$ and the infimum is taken over all such $\sigma$.

Proof of Theorem 1. Let $\mathcal{E}=(A, C)$ be a condenser, where $A=\left\{z \in D:\left|z-z_{0}\right|<t+\Delta t\right\}$, $C=\left\{z \in D:\left|z-z_{0}\right| \leqslant t\right\}, t+\Delta t<d_{0}$. Then $f \mathcal{E}=(f A, f C)$ is an annular condenser in $D^{\prime}$ and according to (7) we have the identity

$$
\operatorname{cap}_{p} f \mathcal{E}=\mathcal{M}_{p}(\Delta(\partial f A, \partial f C ; f(A \backslash C)) .
$$

By inequality (8) we obtain

$$
\operatorname{cap}_{p} f \mathcal{E} \geqslant \frac{[\inf l(\sigma)]^{p}}{|f A \backslash f C|^{p-1}} .
$$

Here $l(\sigma)$ is the length of a smooth (infinitely differentiable) curve $\sigma$ being the boundary $\sigma=\partial U$ of a bounded open set $U$ containing $C$ and contained together with its closure $\bar{U}$ in $A$ and the infimum is taken over all such $\sigma$.

On the other hand, by the definition of $Q$-homemorphism w.r.t. $p$-modulus we have

$$
\operatorname{cap}_{p} f \mathcal{E} \leqslant \int_{D} Q(z) \varrho^{p}(z) d m(z)
$$

for each $\varrho \in \operatorname{adm} \Delta(\partial A, \partial C ; A \backslash C)$.

It is easy to check that the function

$$
\varrho(z)=\left\{\begin{aligned}
\frac{1}{\left|z-z_{0}\right| \ln \frac{t+\Delta t}{t},} & z \in A \backslash C \\
0, & z \notin A \backslash C
\end{aligned}\right.
$$

is admissible for the family $\Delta(\partial A, \partial C ; A \backslash C)$ and hence,

$$
\operatorname{cap}_{p} f \mathcal{E} \leqslant \frac{1}{\ln ^{p}\left(\frac{t+\Delta t}{t}\right)} \int_{R} \frac{Q(z)}{\left|z-z_{0}\right|^{p}} d m(z)
$$

where $R=\left\{z \in D: t \leqslant\left|z-z_{0}\right| \leqslant t+\Delta t\right\}$.

Combining inequalities (10) and $(12)$, we get

$$
\frac{[\inf l(\sigma)]^{p}}{|f A \backslash f C|^{p-1}} \leqslant \frac{1}{\ln ^{p}\left(\frac{t+\Delta t}{t}\right)} \int_{R} \frac{Q(z)}{\left|z-z_{0}\right|^{p}} d m(z) .
$$

By the Fubini theorem we have

$$
\int_{R} \frac{Q(z)}{\left|z-z_{0}\right|^{p}} d m(z)=\int_{t}^{t+\Delta t} \frac{d \tau}{\tau^{p}} \int_{S\left(z_{0}, \tau\right)} Q(z)|d z|=2 \pi \int_{t}^{t+\Delta t} \tau^{1-p} q_{z_{0}}(\tau) d \tau
$$


where $q_{z_{0}}(\tau)=\frac{1}{2 \pi \tau} \underset{S\left(z_{0}, \tau\right)}{\int} Q(z)|d z|$ and $S\left(z_{0}, \tau\right)=\left\{z \in \mathbb{C}:\left|z-z_{0}\right|=\tau\right\}$. Thus,

$$
\inf l(\sigma) \leqslant(2 \pi)^{\frac{1}{p}} \frac{|f A \backslash f C|^{\frac{p-1}{p}}}{\ln \left(\frac{t+\Delta t}{t}\right)}\left[\int_{t}^{t+\Delta t} \tau^{1-p} q_{z_{0}}(\tau) d \tau\right]^{\frac{1}{p}}
$$

Employing the isoperimetric inequality

$$
\inf l(\sigma) \geqslant 2 \sqrt{\pi|f C|}
$$

we obtain

$$
2 \sqrt{\pi|f C|} \leqslant(2 \pi)^{\frac{1}{p}} \frac{|f A \backslash f C|^{\frac{p-1}{p}}}{\ln \left(\frac{t+\Delta t}{t}\right)}\left[\int_{t}^{t+\Delta t} \tau^{1-p} q_{z_{0}}(\tau) d \tau\right]^{\frac{1}{p}} .
$$

We introduce a function $\Phi(t)$ for this homeomorphism $f$ as follows:

$$
\Phi(t)=\left|f B\left(z_{0}, t\right)\right|,
$$

where $B\left(z_{0}, t\right)=\left\{z \in \mathbb{C}:\left|z-z_{0}\right| \leqslant t\right\}$. Then it follows from (17) that

$$
2 \sqrt{\pi \Phi(t)} \leqslant(2 \pi)^{\frac{1}{p}} \frac{\left[\frac{\Phi(t+\Delta t)-\Phi(t)}{\Delta t}\right]^{\frac{p-1}{p}}}{\frac{\ln (t+\Delta t)-\ln t}{\Delta t}}\left[\frac{1}{\Delta t} \int_{t}^{t+\Delta t} \tau^{1-p} q_{z_{0}}(\tau) d \tau\right]^{\frac{1}{p}} .
$$

Letting $\Delta t \rightarrow 0$ in inequality $(19)$ and taking into consideration a monotonous increasing of the function $\Phi$ in $t \in\left(0, d_{0}\right)$, for almost all $t$ we have:

$$
\frac{2 \pi^{\frac{p-2}{2(p-1)}}}{t^{\frac{1}{p-1}} q_{z_{0}}^{\frac{1}{p-1}}(t)} \leqslant \frac{\Phi^{\prime}(t)}{\Phi^{\frac{p}{2(p-1)}}(t)}
$$

This implies easily the following inequality:

$$
\frac{2 \pi^{\frac{p-2}{2(p-1)}}}{t^{\frac{1}{p-1}} q_{z_{0}}^{\frac{1}{p-1}}(t)} \leqslant\left(\frac{\Phi^{\frac{p-2}{2(p-1)}}(t)}{\frac{p-2}{2(p-1)}}\right)^{\prime} .
$$

Since $p>2$, the function

$$
g(t)=\frac{\Phi^{\frac{p-2}{2(p-1)}}(t)}{\frac{p-2}{2(p-1)}}
$$

is non-decreasing on $\left(0, d_{0}\right)$, where $d_{0}=\operatorname{dist}\left(z_{0}, \partial D\right)$. Integrating both sides of the inequality in $t \in[\varepsilon, r]$ and taking into consideration that

$$
\int_{\varepsilon}^{r}\left(\frac{\Phi^{\frac{p-2}{2(p-1)}}(t)}{\frac{p-2}{2(p-1)}}\right)^{\prime} d t=\int_{\varepsilon}^{r} g^{\prime}(t) d t \leqslant g(r)-g(\varepsilon) \leqslant \frac{\Phi^{\frac{p-2}{2(p-1)}}(r)-\Phi^{\frac{p-2}{2(p-1)}}(\varepsilon)}{\frac{p-2}{2(p-1)}},
$$

see, for instance, [16, Thm. IV.7.4], we obtain

$$
2 \pi^{\frac{p-2}{2(p-1)}} \int_{\varepsilon}^{r} \frac{d t}{t^{\frac{1}{p-1}} q_{z_{0}}^{\frac{1}{p-1}}(t)} \leqslant \frac{\Phi^{\frac{p-2}{2(p-1)}}(r)-\Phi^{\frac{p-2}{2(p-1)}}(\varepsilon)}{\frac{p-2}{2(p-1)}} .
$$

Letting $\varepsilon \rightarrow 0$ in inequality 23 , we arrive at the estimate

$$
\Phi(r) \geqslant \pi\left(\frac{p-2}{p-1}\right)^{\frac{2(p-1)}{p-2}}\left(\int_{0}^{r} \frac{d t}{t^{\frac{1}{p-1}} q_{z_{0}}^{\frac{1}{p-1}}(t)}\right)^{\frac{2(p-1)}{p-2}} .
$$


Finally, denoting $\Phi(r)=\left|f B\left(z_{0}, r\right)\right|$ in the latter inequality, we get

$$
\left|f B\left(z_{0}, r\right)\right| \geqslant \pi\left(\frac{p-2}{p-1}\right)^{\frac{2(p-1)}{p-2}}\left(\int_{0}^{r} \frac{d t}{t^{\frac{1}{p-1}} q_{z_{0}}^{\frac{1}{p-1}}(t)}\right)^{\frac{2(p-1)}{p-2}}
$$

and this completes the proof of Theorem 1.

\section{Corollaries of Theorem 1}

Theorem 1 implies the following statements.

Employing the condition $q_{z_{0}}(t) \leqslant q_{0} t^{-\alpha}$, we estimate the right hand side of inequality (4) and after elementary transformations we arrive at the following result.

Corollary 1. Let $D$ and $D^{\prime}$ be bounded domains in $\mathbb{C}$ and $f: D \rightarrow D^{\prime}$ be a Q-homeomorphism w.r.t. $p$-modulus as $p>2$. Assume that the function $Q$ satisfies the condition

$$
q_{z_{0}}(t) \leqslant q_{0} t^{-\alpha}, q_{0} \in(0, \infty), \alpha \in[0, \infty)
$$

for $z_{0} \in D$ and almost all $t \in\left(0, d_{0}\right), d_{0}=\operatorname{dist}\left(z_{0}, \partial D\right)$. Then for each $r \in\left(0, d_{0}\right)$ the estimate

$$
\left|f B\left(z_{0}, r\right)\right| \geqslant \pi^{-\frac{\alpha}{p-2}}\left(\frac{p-2}{\alpha+p-2}\right)^{\frac{2(p-1)}{p-2}} q_{0}^{\frac{2}{2-p}}\left|B\left(z_{0}, r\right)\right|^{1+\frac{\alpha}{p-2}}
$$

holds true.

In particular, letting here $\alpha=0$, we obtain the following conclusion.

Corollary 2. Let $D$ and $D^{\prime}$ be bounded domains in $\mathbb{C}$ and $f: D \rightarrow D^{\prime}$ be a Q-homeomorphism w.r.t. $p$-modulus as $p>2$ and $q_{z_{0}}(t) \leqslant q_{0}<\infty$ for almost each $t \in\left(0, d_{0}\right)$, $d_{0}=\operatorname{dist}\left(z_{0}, \partial D\right)$. Then the estimate

$$
\left|f B\left(z_{0}, r\right)\right| \geqslant q_{0}^{\frac{2}{2-p}}\left|B\left(z_{0}, r\right)\right|
$$

holds true for each $r \in\left(0, d_{0}\right)$.

Corollary 3. Suppose that the assumptions of Theorem 1 are satisfied and $Q(z) \leqslant K<\infty$ for almost each $z \in D$. Then the estimate

$$
\left|f B\left(z_{0}, r\right)\right| \geqslant K^{\frac{2}{2-p}}\left|B\left(z_{0}, r\right)\right|
$$

holds true for each $r \in\left(0, d_{0}\right)$.

Remark 1. Corollary 3 is a particular result by Gehring for $E=B\left(z_{0}, r\right)$, see [12, Lm. 7].

Corollary 4. Let $f: \mathbb{B} \rightarrow \mathbb{B}$ be a Q-homeomorphism w.r.t. p-modulus as $p>2$. Assume that the function $Q(z)$ satisfies the condition

$$
q(t) \leqslant \frac{q_{0}}{t \ln ^{p-1} \frac{1}{t}}, q_{0} \in(0, \infty)
$$

for almost each $t \in(0,1)$, where $q(t)=\frac{1}{2 \pi t} \int_{S_{t}} Q(z)|d z|$ is the integral mean over the circumference $S_{t}=\{z \in \mathbb{C}:|z|=t\}$. Then for each $r \in(0,1)$ the estimate

$$
\left|f B_{r}\right| \geqslant \pi\left(\frac{p-2}{p-1}\right)^{\frac{2(p-1)}{p-2}} q_{0}^{\frac{2}{2-p}}\left(r \ln \frac{e}{r}\right)^{\frac{2(p-1)}{p-2}}
$$

holds true, where $B_{r}=\{z \in \mathbb{C}:|z| \leqslant r\}$. 


\section{EXTREMAL PROBLEMS FOR AREA FUNCTIONAL}

Let $Q: \mathbb{B} \rightarrow[0, \infty]$ be a measurable function satisfying the condition

$$
q(t) \leqslant q_{0}, q_{0} \in(0, \infty)
$$

for almost each $t \in(0,1)$, where $q(t)=\frac{1}{2 \pi t} \int_{S_{t}} Q(z)|d z|$ is the integral mean over the circumference $S_{t}=\{z \in \mathbb{C}:|z|=t\}$.

Let $\mathcal{H}=\mathcal{H}\left(q_{0}, p, \mathbb{B}\right)$ be the set of all $Q$-homeomorphisms $f: \mathbb{B} \rightarrow \mathbb{C}$ w.r.t. $p$-modulus as $p>2$ obeying condition (32). On the class $\mathcal{H}$ we consider the area functional

$$
\mathbf{S}_{r}(f)=\left|f B_{r}\right| .
$$

Theorem 2. For each $r \in[0,1]$ the identity

$$
\min _{f \in \mathcal{H}} \mathbf{S}_{r}(f)=\pi q_{0}^{\frac{2}{2-p}} r^{2}
$$

holds true.

Proof. Corollary 2 implies immediately the estimate

$$
\mathbf{S}_{r}(f) \geqslant \pi q_{0}^{\frac{2}{2-p}} r^{2}
$$

Let us specify a homeomorphism $f \in \mathcal{H}$, at which the minimum of the functional $\mathbf{S}_{r}(f)$ is attained. Let $f_{0}: \mathbb{B} \rightarrow \mathbb{C}$, where

$$
f_{0}(z)=q_{0}^{\frac{1}{2-p}} z
$$

It is obvious that (35) becomes the identity at the mapping $f_{0}$. It remains to show that the mapping defined in such way is a $Q$-homemorphism w.r.t. $p$-modulus with $Q(z)=q_{0}$. Indeed,

$$
l\left(z, f_{0}\right)=L\left(z, f_{0}\right)=q_{0}^{\frac{1}{2-p}}, \quad J\left(z, f_{0}\right)=q_{0}^{\frac{2}{2-p}}
$$

and

$$
K_{I, p}\left(z, f_{0}\right)=\frac{J\left(z, f_{0}\right)}{l^{p}\left(z, f_{0}\right)}=q_{0} .
$$

By Theorem 1.1 in work [17], the mapping $f_{0}$ is a $Q$-homeomorphism w.r.t. $p$-modulus with $Q(z)=K_{I, p}\left(z, f_{0}\right)=q_{0}$.

\section{BIBLIOGRAPHY}

1. B.V. Bojarski. Homeomorhpic solutions to Beltrami systems // Dokl. Akad. Nauk SSSR. 102, 661-664 (1955). (in Russian).

2. F.W. Gehring, E. Reich. Area distortion under quasiconformal mappings // Ann. Acad. Sci. Fenn. Ser. A I Math. 388, 15 pp. (1966).

3. K. Astala. Area dislortron of quasrconformal mapprngs // Acta Math. 173:1, 37-60 (1994).

4. A. Eremenko, D.H. Hamilton. On the area distortion by quasiconformal mappings // Proc. Amer. Math. Soc. 123:9, 2793-2797 (1995).

5. M.A. Lavrent'ev. Variational methods for boundary value problems for systems of elliptic equations. Nauka, Moscow (1962). [P. Noordhoff Ltd, Groningen (1963).]

6. B. Bojarski, V. Gutlyanskii, O. Martio, V. Ryazanov. Infinitesimal geometry of quasiconformal and bi-Lipschitz mappings in the plane. EMS Tracts in Math. 19. EMS Publishing House, Zürich (2013).

7. T.V. Lomako, R.R. Salimov. To the theory of extremal problems // in Collection of the Inst. Math. NAS Ukraine. 7:2, 264-269 (2010). (in Russian).

8. R.R. Salimov. Lower estimates of p-modulus and mappings of Sobolev's class // Algeb. Anal. 26:6, 143-171 (2014). [St. Petersburg Math. J. 26:6, 965-984 (2015).] 
9. L.V. Ahlfors. Lectures on quasiconformal mappings. D. Van Nostrand Company, Princeton (1966).

10. O. Lehto, K. Virtanen. Quasiconformal mappings in the plane Springer, New York (1973).

11. C.J. Bishop, V.Ya. Gutlyanskii, O. Martio, M. Vuorinen On conformal dilatation in space // Intern. J. Math. Math. Sci. 2003:22, 1397-1420 (2003).

12. F.W. Gehring. Lipschitz mappings and the p-capacity of ring in $n$-space // in "Advances in the theory of Riemann surfaces", Proc. Conf. Stonybrook, N.Y. 175-193 (1971).

13. O. Martio, S. Rickman, J. Väisälä. Definitions for quasiregular mappings // Ann. Acad. Sci. Fenn. Ser. A1. Math. 448, 40 pp. (1969).

14. V.A. Shlyk. The equality between p-capacity and p-modulus // Sibir. Matem. Zhurn. 34:56, 216-221 (1993). [Siber. Math. J. 34:56, 1196-1120 (1993).]

15. V.I. Kruglikov. Capacity of condensers and spatial mappings quasiconformal in the mean // Matem. Sborn. 130:2, 185-206 (1986). [Math. USSR-Sb. 58:1, 185-205 (1987).]

16. S. Saks. Theory of the integral. G. E. Stechert \& Co., New York (1937).

17. R. Salimov, E. Sevost'yanov. The Poletskii and Vaisala inequalities for the mappings with (p,q)distortion // Compl. Variab. Ellip. Equat. 599:2, 217-231 (2014).

Bogdan Anatol'evich Klishchuk,

Institute of Mathematics,

National Academy of Sciences of Ukraine,

Tereschenkivska str. 3,

01601, Kiev, Ukraine

E-mail: bogdanklishchuk@mail.ru

Ruslan Radikovich Salimov,

Institute of Mathematics,

National Academy of Sciences of Ukraine,

Tereschenkivska str. 3,

01601, Kiev, Ukraine

E-mail: ruslan623@yandex.ru 